\title{
The Japanese Space Gravitational Wave Antenna; DECIGO
}

Seiji Kawamura $^{1}$, Masaki Ando ${ }^{2}$, Takashi Nakamura ${ }^{3}$, Kimio Tsubono ${ }^{2}$, Takahiro Tanaka ${ }^{3}$, Ikkoh Funaki ${ }^{4}$, Naoki Seto ${ }^{1}$, Kenji Numata ${ }^{5}$, Shuichi Sato ${ }^{1}$, Kunihito Ioka ${ }^{6}$, Nobuyuki Kanda ${ }^{7}$, Takeshi Takashima ${ }^{4}$, Kazuhiro Agatsuma ${ }^{2}$, Tomotada Akutsu' ${ }^{2}$, Tomomi Akutsu ${ }^{2}$, Koh-suke Aoyanagi ${ }^{8}$, Koji Arai ${ }^{1}$, Yuta Arase $^{2}$, Akito Araya ${ }^{9}$, Hideki Asada ${ }^{10}$, Yoichi Aso ${ }^{11}$, Takeshi Chiba ${ }^{12}$, Toshikazu Ebisuzaki $^{13}$, Motohiro Enoki ${ }^{14}$, Yoshiharu Eriguchi ${ }^{15}$, Masa-Katsu Fujimoto ${ }^{1}$, Ryuichi Fujita $^{16}$, Mitsuhiro Fukushima ${ }^{1}$, Toshifumi Futamase ${ }^{17}$, Katsuhiko Ganzu $^{3}$, Tomohiro Harada ${ }^{18}$, Tatsuaki Hashimoto ${ }^{4}$, Kazuhiro Hayama ${ }^{19}$, Wataru Hikida $^{16}$, Yoshiaki Himemoto ${ }^{20}$, Hisashi Hirabayashi ${ }^{21}$, Takashi Hiramatsu ${ }^{2}$, Feng-Lei Hong ${ }^{22}$, Hideyuki Horisawa ${ }^{23}$, Mizuhiko Hosokawa ${ }^{24}$, Kiyotomo Ichiki ${ }^{2}$, Takeshi Ikegami $^{22}$, Kaiki T. Inoue ${ }^{25}$, Koji Ishidoshiro ${ }^{2}$, Hideki Ishihara ${ }^{7}$, Takehiko Ishikawa $^{26}$, Hideharu Ishizaki ${ }^{1}$, Hiroyuki Ito ${ }^{24}$, Yousuke Itoh ${ }^{27}$, Shogo Kamagasako $^{2}$, Nobuki Kawashima ${ }^{25}$, Fumiko Kawazoe ${ }^{28}$, Hiroyuki Kirihara ${ }^{2}$, Naoko Kishimoto ${ }^{4}$, Kenta Kiuchi ${ }^{8}$, Shiho Kobayashi ${ }^{29}$, Kazunori Kohri ${ }^{30}$, Hiroyuki Koizumi ${ }^{2}$, Yasufumi Kojima ${ }^{31}$, Keiko Kokeyama ${ }^{28}$, Wataru Kokuyama $^{2}$, Kei Kotake', Yoshihide Kozai ${ }^{32}$, Hideaki Kudoh ${ }^{2}$, Hiroo Kunimori ${ }^{33}$, Hitoshi Kuninaka ${ }^{4}$, Kazuaki Kuroda ${ }^{34}$, Kei-ichi Maeda ${ }^{8}$, Hideo Matsuhara ${ }^{4}$, Yasushi Mino $^{35}$, Osamu Miyakawa ${ }^{35}$, Shinji Miyoki ${ }^{34}$, Mutsuko Y. Morimoto ${ }^{4}$, Tomoko Morioka ${ }^{2}$, Toshiyuki Morisawa ${ }^{3}$, Shigenori Moriwaki ${ }^{36}$, Shinji Mukohyama $^{2}$, Mitsuru Musha ${ }^{37}$, Shigeo Nagano ${ }^{24}$, Isao Naito ${ }^{38}$, Noriyasu Nakagawa $^{2}$, Kouji Nakamura ${ }^{1}$, Hiroyuki Nakano ${ }^{39}$, Kenichi Nakao ${ }^{7}$, Shinichi Nakasuka $^{2}$, Yoshinori Nakayama ${ }^{40}$, Erina Nishida ${ }^{28}$, Kazutaka Nishiyama ${ }^{4}$, Atsushi Nishizawa ${ }^{41}$, Yoshito Niwa ${ }^{41}$, Masatake Ohashi ${ }^{34}$, Naoko Ohishi ${ }^{1}$, Masashi Ohkawa $^{42}$, Akira Okutomi ${ }^{2}$, Kouji Onozato ${ }^{2}$, Kenichi Oohara ${ }^{42}$, Norichika Sago ${ }^{43}$, Motoyuki Saijo $^{43}$, Masaaki Sakagami ${ }^{41}$, Shin-ichiro Sakai ${ }^{4}$, Shihori Sakata ${ }^{28}$, Misao Sasaki $^{44}$, Takashi Sato ${ }^{42}$, Masaru Shibata ${ }^{15}$, Hisaaki Shinkai ${ }^{45}$, Kentaro Somiya $^{46}$, Hajime Sotani ${ }^{47}$, Naoshi Sugiyama ${ }^{48}$, Yudai Suwa ${ }^{2}$, Hideyuki Tagoshi ${ }^{16}$, Kakeru Takahashi ${ }^{2}$, Keitaro Takahashi ${ }^{44}$, Tadayuki Takahashi ${ }^{4}$, Hirotaka Takahashi $^{49}$, Ryuichi Takahashi ${ }^{48}$, Ryutaro Takahashi ${ }^{1}$, Akiteru Takamori ${ }^{9}$, Tadashi Takano $^{4}$, Keisuke Taniguchi ${ }^{50}$, Atsushi Taruya ${ }^{2}$, Hiroyuki Tashiro ${ }^{3}$, Mitsuru Tokuda ${ }^{7}$, Masao Tokunari ${ }^{2}$, Morio Toyoshima ${ }^{24}$, Shinji Tsujikawa ${ }^{51}$, Yoshiki Tsunesada ${ }^{52}$, Ken-ichi Ueda ${ }^{37}$, Masayoshi Utashima ${ }^{53}$, Hiroshi Yamakawa $^{54}$, Kazuhiro Yamamoto ${ }^{1}$, Toshitaka Yamazaki ${ }^{1}$, Jun'ichi Yokoyama ${ }^{2}$, Chul-Moon Yoo ${ }^{44}$, Shijun Yoshida ${ }^{17}$, Taizoh Yoshino ${ }^{55}$

${ }^{1}$ National Astronomical Observatory of Japan, Mitaka, Tokyo, 181-8588, Japan

${ }^{2}$ The University of Tokyo, Bunkyo, Tokyo, 113-0033, Japan

${ }^{3}$ Kyoto University, Kyoto, Kyoto, 606-8502, Japan

${ }^{4}$ Institute of Space and Astronautical Science, Japan Aerospace Exploration Agency,

Sagamihara, Kanagawa, 229-8510, Japan

${ }^{5}$ NASA Goddard Space Flight Center, Greenbelt, MD 20771, USA

${ }^{6}$ High Energy Accelerator Research Organization, Tsukuba, Ibaraki, 305-0801, Japan 
${ }^{7}$ Osaka City University, Osaka, Osaka, 558-8585, Japan

${ }^{8}$ Waseda University, Shinjuku, Tokyo, 169-8555, Japan

${ }^{9}$ Earthquake Research Institute, The University of Tokyo, Bunkyo, Tokyo, 113-0032, Japan

${ }^{10}$ Hirosaki University, Hirosaki, Aomori, 036-8560, Japan

${ }^{11}$ Columbia University, New York, NY 10027, USA

${ }^{12}$ Nihon University, Setagaya, Tokyo, 156-8550, Japan

${ }^{13}$ RIKEN, Wako, Saitama, 351-0198, Japan

${ }^{14}$ Tokyo Keizai University, Kokubunji, Tokyo, 185-8502, Japan

${ }^{15}$ The University of Tokyo, Meguro, Tokyo, 153-8902, Japan

${ }^{16}$ Osaka University, Toyonaka, Osaka, 560-0043, Japan

${ }^{17}$ Tohoku University, Sendai, Miyagi, 980-8578, Japan

${ }^{18}$ Rikkyo University, Toshima, Tokyo, 171-8501, Japan

${ }^{19}$ University of Texas, Brownsville, Texas, 78520, USA

${ }^{20}$ Shibaura Institute of Technology, Saitama, Saitama, 337-8570, Japan

${ }^{21}$ Space Educations Center, Japan Aerospace Exploration Agency, Sagamihara, Kanagawa, 229-8510, Japan

${ }^{22}$ National Institute of Advanced Industrial Science and Technology, Tsukuba, Ibaraki, 305-8563, Japan

${ }^{23}$ Tokai University, Hiratsuka, Kanagawa, 259-1292, Japan

${ }^{24}$ National Institute of Information and Communications Technology, Koganei, Tokyo, 184-8795, Japan

${ }^{25}$ Kinki University, Higashi-Osaka, Osaka, 577-8502, Japan

${ }^{26}$ Institute of Space and Astronautical Science, Japan Aerospace Exploration Agency, Tsukuba, Ibaraki, 305-8505, Japan

${ }^{27}$ University of Wisconsin - Milwaukee, Milwaukee, WI 53201-0413, USA

${ }^{28}$ Ochanomizu University, Bunkyo, Tokyo, 112-8610, Japan

${ }^{29}$ Astrophysics Research Institute, Liverpool John Moores University, Egerton Wharf, Birkenhead L41 1LD, UK

${ }^{30}$ Lancaster University, LA1 4YB, UK

${ }^{31}$ Hiroshima University, Higashi-hiroshima, Hiroshima, 739-8526, Japan

${ }^{32}$ Gunma Astronomical Observatory, Agatsuma, Gunma, 377-0702, Japan

${ }^{33}$ National Institute of Information and Communications Technology, Bunkyo, Tokyo, 113-0001, Japan

${ }^{34}$ Institute for Cosmic Ray Research, The University of Tokyo, Kashiwa, Chiba, 2778582, Japan

${ }^{35}$ California Institute of Technology, Pasadena, CA 91125, USA

${ }^{36}$ The University of Tokyo, Kashiwa, Chiba, 277-8561, Japan

${ }^{37}$ Institute for Laser Science, The University of Electro-Communications, Chofu, Tokyo, 182-8585, Japan

${ }^{38}$ Numakage, Saitama, Saitama, 336-0027, Japan

${ }^{39}$ Rochester Institute of Technology, Rochester, NY 14623, USA

${ }^{40}$ National Defense Academy, Yokosuka, Kanagawa, 239-8686, Japan

${ }^{41}$ Kyoto University, Kyoto, Kyoto, 606-8501, Japan

${ }^{42}$ Niigata University, Niigata, Niigata, 950-2181, Japan

${ }^{43}$ University of Southampton, Southampton SO17 1BJ, UK

${ }^{44}$ Yukawa Institute for Theoretical Physics, Kyoto University, Kyoto, Kyoto, 6068502, Japan

${ }^{45}$ Osaka Institute of Technology, Hirakata, Osaka, 573-0196, Japan

${ }^{46}$ Albert Einstein Institute, Max Planck Institute for Gravitational Physics, D-14476

Potsdam, Germany 
${ }^{47}$ Aristotle University of Thessaloniki, Thessaloniki, 54124, Greece

${ }^{48}$ Nagoya University, Nagoya, Aichi, 464-8602, Japan

${ }^{49}$ Nagaoka University of Technology, Nagaoka, Niigata, 940-2188, Japan

${ }^{50}$ University of Illinois at Urbana-Champaign, Urbana, IL 61801, USA

${ }^{51}$ Gunma National College of Technology, Maebashi, Gunma, 371-8530, Japan

${ }^{52}$ Tokyo Institute of Technology, Ookayama, Meguro-ku, Tokyo, 152-8550, Japan

${ }_{53}^{53}$ Japan Aerospace Exploration Agency, Tsukuba, Ibaraki, 305-8505, Japan

${ }^{54}$ Research Institute for Sustainable Humanosphere, Kyoto University, Uji, Kyoto, 611-0011

${ }^{55}$ Nakamura-minami, Nerima, Tokyo, 176-0025, Japan

* Paper presented at the conference by Seiji Kawamura

E-mail address: seiji.kawamura@nao.ac.jp

\begin{abstract}
DECi-hertz Interferometer Gravitational wave Observatory (DECIGO) is the future Japanese space gravitational wave antenna. DECIGO is expected to open a new window of observation for gravitational wave astronomy especially between $0.1 \mathrm{~Hz}$ and $10 \mathrm{~Hz}$, revealing various mysteries of the universe such as dark energy, formation mechanism of supermassive black holes, and inflation of the universe. The pre-conceptual design of DECIGO consists of three drag-free spacecraft, whose relative displacements are measured by a differential FabryPerot Michelson interferometer. We plan to launch two missions, DECIGO pathfinder and preDECIGO first and finally DECIGO in 2024.
\end{abstract}

\title{
1. Space gravitational wave antenna DECIGO
}

DECIGO is the future Japanese space gravitational wave antenna. It stands for DECi-hertz Interferometer Gravitational wave Observatory [1][2]. The objectives of DECIGO are to detect gravitational waves from various kinds of sources mainly between $0.1 \mathrm{~Hz}$ and $10 \mathrm{~Hz}$ and open a new window of observation for gravitational wave astronomy.

DECIGO will bridge the frequency gap between LISA [3] and terrestrial detectors such as LCGT [4]. It can play a role of follow-up for LISA by observing inspiral sources that have moved above the LISA band, and can also play a role of predictor for terrestrial detectors by observing inspiral sources that have not yet moved into the terrestrial detector band.

DECIGO can reach an extremely good sensitivity. This is because the confusion limiting noise caused by irresolvable gravitational wave signals from many compact binaries is expected to be very low above $0.1 \mathrm{~Hz}[5]$.

\section{Pre-conceptual design of DECIGO}

As shown in Fig. 1, the pre-conceptual design of DECIGO consists of three drag-free spacecraft, whose relative displacements are measured by a differential Fabry-Perot (FP) Michelson interferometer. The arm length was chosen to be $1,000 \mathrm{~km}$ in order to realize a finesse of 10 with a 1 $\mathrm{m}$ diameter mirror and $0.5 \mu \mathrm{m}$ laser light. The mass of the mirror is $100 \mathrm{~kg}$ and the laser power is 10 $\mathrm{W}$. Three sets of such interferometers sharing the mirrors as arm cavities comprise one cluster of DECIGO. The constellation of DECIGO is composed of four clusters of DECIGO located separately in the heliocentric orbit with two of them nearly at the same position.

The FP configuration requires the distance between two mirrors, thus, the distance between two spacecraft to be constant during continuous operations. This makes DECIGO very different from a possible counterpart with the transponder-type detector (e.g. LISA), where the spacecraft, which are much farther apart, are freely falling according to their local gravitational field. We adopted the FP configuration because it can provide a better shot-noise-limited sensitivity than the transponder configuration due to the enhanced gravitational wave signals. 


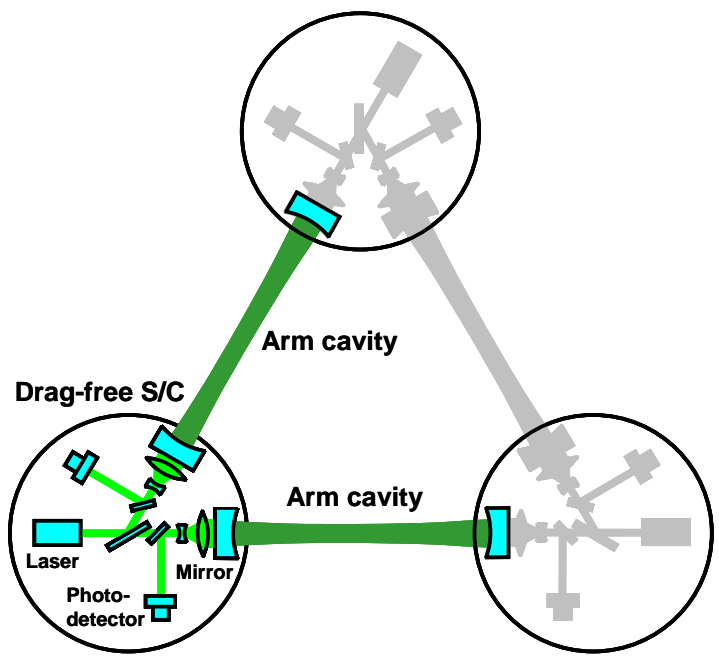

Fig.1. Pre-conceptual design of DECIGO.

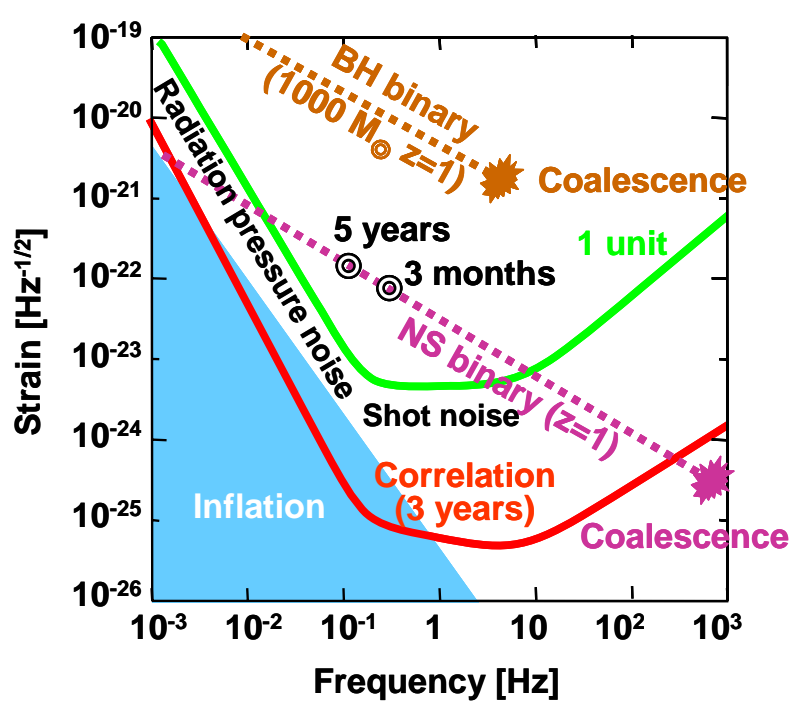

Fig. 2. Sensitivity goal of DECIGO and expected gravitational wave signals.

The FP configuration requires an additional system beyond that of an ordinary drag-free system. In the ordinary drag-free system, the outer spacecraft simply follows the motion of the mirror inside. However, in the FP configuration, the distance between the mirrors should be kept constant, which requires that one of the two mirrors should be controlled with the other mirror as a reference. As a result, the reference mirror dictates the motion of the other mirror by the FP interferometer control system as well as both spacecraft by the drag-free control system. It should be also noted that the FP interferometer control signals, which include gravitational wave signals, are not contaminated by the noisy drag-free control signals.

The lock acquisition of the FP arm cavity is a challenging task. In a ground-based interferometer, the relative motion of the two mirrors of a cavity is small enough to acquire lock of the cavity without much difficulty because the suspension systems of the mirrors are virtually connected to the ground at zero frequency. However, the relative motion of the two spacecraft in space is expected to be much higher. Therefore, we need an additional system that detects the relative motion of the mirrors, and gradually reduces it by actuating the mirrors. Once the relative motion of the mirrors is suppressed well enough, the lock acquisition of the FP cavity will be straightforward.

\section{Sensitivity goal of DECIGO and science obtained by DECIGO}

The ideal sensitivity of DECIGO is limited only by quantum noise, as shown in Fig. 2. The sensitivity is limited by the radiation pressure noise below $0.15 \mathrm{~Hz}$, and it has an $f^{-2}$ frequency dependence. The shot noise limits the sensitivity above $0.15 \mathrm{~Hz}$. It is flat up to $7.5 \mathrm{~Hz}$, and above $7.5 \mathrm{~Hz}$ it increases in proportion with frequency because of the signal cancellation in the arm cavities.

In order to realize the sensitivity goal of DECIGO, all the practical noise should be suppressed well below this level. This imposes stringent requirements for the subsystems of DECIGO. We anticipate that extremely rigorous investigations are required to attain the requirements especially in the acceleration noise and frequency noise.

Nevertheless, accomplishing the sensitivity goal of DECIGO will ensure a variety of fruitful sciences to be obtained.

\section{(1) Characterization of dark energy}

DECIGO can detect gravitational waves coming from neutron star binaries at $\mathrm{z}=1$ for five years prior to coalescences. It is expected that within this range about 7,000 neutron star binaries will coalesce every year. Therefore, DECIGO will detect gravitational waves coming from a large number 
of neutron star binaries at the same time. By analyzing the waveforms of these gravitational wave signals precisely, it is possible to determine the acceleration of the expansion of the universe [1]. The acceleration of the expansion of the universe can be also measured by finding host galaxies of each binary and determining their red shifts optically [6]. This will lead to better characterization of dark energy.

(2) Formation mechanism of supermassive black holes in the center of galaxies

DECIGO can detect gravitational waves coming from coalescences of intermediate-mass black hole binaries with an extremely high fidelity. For example the coalescences of black hole binaries of 1,000 solar masses at $\mathrm{z}=1$ give a signal to noise ratio of 6,000 . This will make it possible to collect numerous data about the relationship between the mass of the black holes and the frequency of the coalescences, which will reveal the formation mechanism of supermassive black holes in the center of galaxies.

\section{(3) Verification and characterization of inflation}

DECIGO can detect stochastic background corresponding to $\Omega_{\mathrm{GW}}=2 \times 10^{-16}$ by correlating the data from the two clusters of DECIGO for three years. According to the standard inflation model, it is expected that we could detect gravitational waves produced at the inflation period of the universe with DECIGO. This is extremely significant because gravitational waves are the only means which make it possible to directly observe the inflation of the universe.

While the inflation background is the primary target for the correlation analysis with the two clusters, it would be important to carefully design the system so that we can disclose various aspects of stochastic gravitational wave backgrounds. One of the interesting measures from fundamental physics is the Stokes V parameter. This parameter characterizes the asymmetry of the amplitudes of the rightand left-handed waves, and it is a powerful measure to probe violation of parity symmetry that interchanges the two circular-polarization modes. By slightly adjusting the relative configuration of the two clusters, we can set sensitivity to the Stokes V parameter [7].

\section{Roadmap to DECIGO}

DECIGO pathfinder (DPF) and pre-DECIGO will be launched before DECIGO. DPF will test the key technologies with one spacecraft. We expect that it will be launched in 2012. Pre-DECIGO is supposed to detect gravitational waves with minimum specifications. We hope that it will be launched in 2018. Finally it is expected that DECIGO will be launched in 2024 to open a new window of observation for gravitational wave astronomy.

\section{Acknowledgment}

This research was partially supported by the Ministry of Education, Science, Sports and Culture, Grant-in-Aid for Scientific Research.

\section{References}

[1] Seto N, Kawamura S and Nakamura T 2001 Possibility of direct measurement of the acceleration of the universe using $0.1 \mathrm{~Hz}$ band laser interferometer gravitational wave antenna in space Phys. Rev. Lett. 87221103

[2] Kawamura S et al 2006 The Japanese Space Gravitational Wave Antenna - DECIGO Class. Quantum Grav. 23 S125

[3] LISA: System and Technology Study Report, ESA document ESA-SCI (2000)

[4] Kuroda K et al 2002 Japanese large-scale interferometers Class. Quantum Grav. 191237

[5] Farmer A J and Phinney E S 2003 The gravitational wave background from cosmological compact binaries Mon. Not. R. Astron. Soc. 3461197

[6] Schutz B F 1986 Determining the Hubble constant from gravitational wave observations Nature 323310

[7] Seto N 2007 Quest for circular polarization of a gravitational wave background and orbits of laser interferometers in space Phys. Rev. D 75061302 